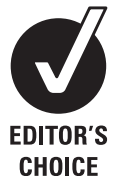

CHOICE
${ }^{1}$ Division of Public Health and Primary Health Care, University of Oxford, Oxford, UK

${ }^{2}$ WHO Collaborating Centre for Obesity Prevention, Deakin University, Victoria, Australia ${ }^{3}$ Division of Medicine, Lancaster University, Lancaster, UK

\section{Correspondence to}

Steven Allender, University of Oxford, Rosemary Rue Building, Old Road Campus, Headington, Oxford OX3 7LF, UK; steven.allender@dphpc.ox.ac. uk

Accepted 27 May 2011 Published Online First 28 June 2011

\title{
Relative deprivation between neighbouring wards is predictive of coronary heart disease mortality after adjustment for absolute deprivation of wards
}

\author{
Steven Allender, ${ }^{1}{ }^{1}$ Peter Scarborough, ${ }^{1}$ Thomas Keegan, ${ }^{3}$ Mike Rayner ${ }^{1}$
}

\begin{abstract}
Background The aims of this study were to assess whether deprivation inequality at small area level in England is associated with coronary heart disease (CHD) mortality rates and to assess whether this provides evidence of an association between area-level and individual-level risk.
\end{abstract}

Methods Mortality rates for all wards in England were calculated using all CHD deaths between 2001 and 2006. Ward-level deprivation was measured using the Carstairs Index. Deprivation inequality within local authorities (LAs) was measured by the IQR of deprivation for wards within the LA. Relative deprivation for wards was measured as the modulus of the difference between deprivation for the ward and average deprivation for all neighbouring wards.

Results Deprivation inequality within LAs was positively associated with CHD mortality rates per 100000 (eg, all men $\beta ; 95 \% \mathrm{Cl}=2.7 ; 1.1$ to 4.3 ) after adjustment for absolute deprivation ( $p<0.001$ for all models). Relative deprivation for wards was positively associated with CHD mortality rates per 100000 (eg, all men 1.4; 0.7 to 2.1) after adjustment for absolute deprivation $(p<0.001$ for all models). Subgroup analyses showed that relative deprivation was independently associated with $\mathrm{CHD}$ mortality rates in both affluent and deprived wards. Conclusions Rich wards surrounded by poor areas have higher CHD mortality rates than rich wards surrounded by rich areas, and poor wards surrounded by rich areas have worse $\mathrm{CHD}$ mortality rates than poor wards surrounded by poor areas. Local deprivation inequality has a similar adverse impact on both rich and poor areas, supporting the hypothesis that income inequality of an area has an impact on individual-level health outcomes.

\section{INTRODUCTION}

Social position has a large and well-documented impact on many health outcomes, ${ }^{1}$ and it is thought that the mechanisms of this impact include both material deprivation and psychological stress. ${ }^{2}{ }^{3}$ There are two competing hypotheses in the health literature regarding the impact of social position on health outcomes. One hypothesis is that within developed high-income societies, the total amount of social inequality (usually proxied by measures of income inequality or deprivation inequality) within society increases the risk of poor health outcomes for all individuals within the society. ${ }^{2}{ }^{5}$ This hypothesis is supported by analyses of large areas (eg, Organisation for Economic Co-operation and Development countries, US states) that show that area-level health outcomes (such as life expectancy) are more strongly associated with the amount of income inequality within an area than with the mean income of the society ${ }^{6}$ and a meta-analysis of multilevel cohort studies. ${ }^{5}$

The competing hypothesis suggests that an individual's social position is associated with individual-level adverse health outcomes, but the total amount of social inequality within society is not. This hypothesis seems incompatible with area-level results that have shown a relationship between income inequality and rates of adverse health outcomes after adjustment for aggregated income levels, but it has been demonstrated that this relationship may be due to a statistical artefact. ${ }^{7}$ If the relationship between risk of adverse health outcomes and income at the individual level is nonlinear and diminishes as income increases (so that an increase of income of $£ 1000$ per year has a greater impact on reducing risk of adverse health outcome for an individual on a low income than for an individual on a high income), then some relationship between income inequality and health outcomes would be expected at area level, even if there is no relationship between income inequality of a society and risk at the individual level. A third emerging hypothesis based on recent work in the USA has suggested that the association between income inequality and poorer health may be confounded by previously unaccounted-for social and genetic factors, which themselves are associated with income. ${ }^{8} 9$

Outside the USA, evidence for associations between deprivation inequality and poor health in areas smaller than states has not been consistent, and evidence for the same relationship elsewhere has been also been inconsistent. ${ }^{8}$ In contrast, work in the UK, within England, has been shown that all-cause mortality rates for local authorities (LAs) are independently related to both absolute deprivation levels and the amount of deprivation inequality within the LAs, ${ }^{10}$ to which the authors concluded that their results 'support the hypothesis that variations in income contribute an additional effect on mortality over the effect of deprivation alone'. We aimed to reinvestigate this finding using more recent data on coronary heart disease (CHD) mortality rates for all wards and LAs in England. Our research questions were: Is there an association between deprivation inequality at small area level in England and CHD mortality rates? If so, does this association provide evidence to support the hypothesis of an impact of deprivation inequality on individual-level health outcomes? 


\section{METHODS}

The outcomes considered for this paper were CHD mortality rates for all men, all women and for men and women aged $\leq 75$ years (defined throughout as premature mortality). Mortality data were provided by the Office for National Statistics and include all deaths in England between 2001 and 2006 inclusive where the underlying cause of death was recorded as CHD (ICD-10: I20-I25). Mortality rates age standardised to the European Standard Population were constructed using mortality and population estimates by sex, 5-year age band and ward of residence. Population estimates were taken from the 2001 census. $^{11}$

Two levels of geographical area were used for this analysis: LAs and standard table wards (henceforth simply 'wards'). In England, there are 356 LAs and 7929 wards. The deprivation index used was the 2001 Carstairs Index ${ }^{12}{ }^{13}$ drawn from the 2001 census for every ward in England. The index is a sum of $z$ scores for the following area-level variables: \% economically active males who are unemployed, \% overcrowded (more than one person per room) households, \% adults with no access to a car, and \% adults in households with head of household in social class IV or V. Deprivation inequality within LAs was measured by the IOR of the Carstairs Index for all wards within each LA-the same method as applied in the earlier analysis of total mortality rates. ${ }^{10}$ Deprivation inequality could not be applied to two LAs (City of Westminster and the Isles of Scilly) as they only contain one ward; they were not retained for further analysis.

Four sets of LA-level spatial error regressions models were built with CHD mortality rates among men and women, and premature CHD mortality rates among men and women as the dependent variables. The independent variables were absolute deprivation (Carstairs Index for each LA) and deprivation inequality (IOR of Carstairs Index for wards within each LA). Independent variables were included in both univariate and multivariate models.

Further sets of regression models were then built with wardlevel CHD mortality rates as dependent variables. The independent variables were absolute deprivation (Carstairs Index for each ward) and relative deprivation (the modulus of the difference between the Carstairs Index for a ward and the average Carstairs Index of all neighbouring wards). The relative deprivation variable is a continuous measure (bounded at zero) of the difference between deprivation levels in a ward and the average deprivation in all surrounding wards. The variable measures the size of the deprivation inequality between a ward and its neighbours, and as such does not distinguish between a deprived ward surrounded by affluent wards and an affluent ward surrounded by deprived wards. As before, the independent variables were included in both univariate and multivariate models. 'Neighbouring wards' were defined using a queen's firstorder matrix. ${ }^{14}$ Regression models with ward-level female premature $\mathrm{CHD}$ mortality rates as the dependent variables were not constructed as $9 \%$ of wards had zero mortality events, resulting in a non-normal distribution. Subgroup analyses were conducted restricted to affluent wards (all wards with absolute deprivation lower than the median) and deprived wards (all wards with absolute deprivation higher than the median). All statistical analyses were conducted using Stata V.11 software and GeoDa software. ${ }^{15}$

\section{RESULTS}

Table 1 provides descriptive statistics for the population and number of CHD mortalities in LAs in England. Figure 1 demonstrates the strong association between level of deprivation and CHD mortality rates for both sexes, at all ages and premature mortality. Table 2 shows the correlations between the mortality rates, absolute deprivation and deprivation inequality at LA level. All the variables were strongly correlated with each other $(r>0.50)$. The correlation between absolute deprivation and deprivation inequalities was 0.58 , showing that the more deprived LAs were likely to have a greater level of deprivation inequality than the less deprived LAs.

The distribution of the LA-level CHD mortality rates was checked and was found to be reasonably normal and hence could be used as dependent variables in linear regression models. Poisson regression analysis using the number of events in each LA was not attempted as the event counts did not follow a Poisson distribution (results not shown). Table 3 shows the results of the LA-level spatial regression analyses. For each of the outcomes, both absolute deprivation and deprivation inequality were shown to be

Table 1 Descriptive statistics for CHD mortality and deprivation for English local authority areas 2001-2006 (LAs, $n=354 ;$ wards, $n=7927$ )

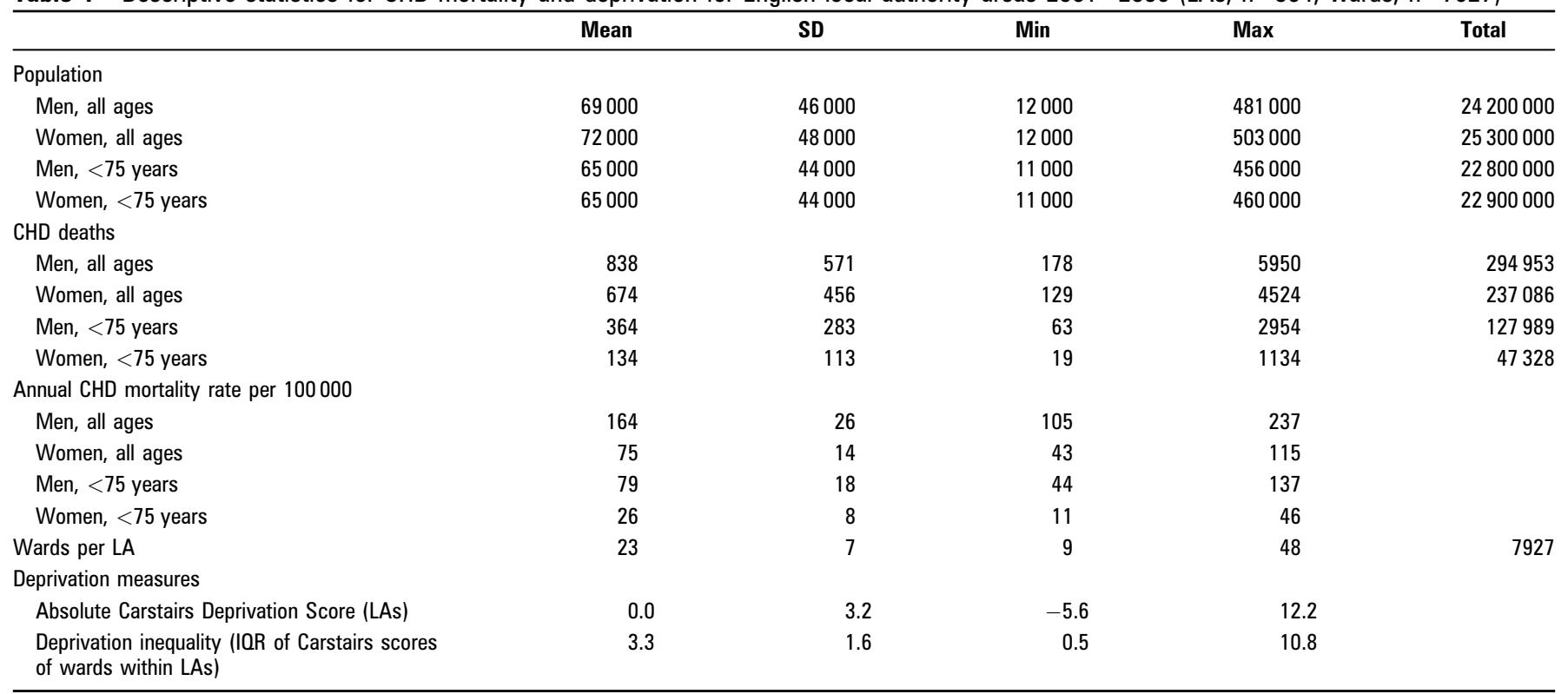

CHD, coronary heart disease; LAs, local authorities. 
Figure 1 Coronary heart disease (CHD) mortality rate $(95 \% \mathrm{Cls})$ for men and women by local authority deprivation quintile, England 2001-2006. Scales differ between charts within this figure.
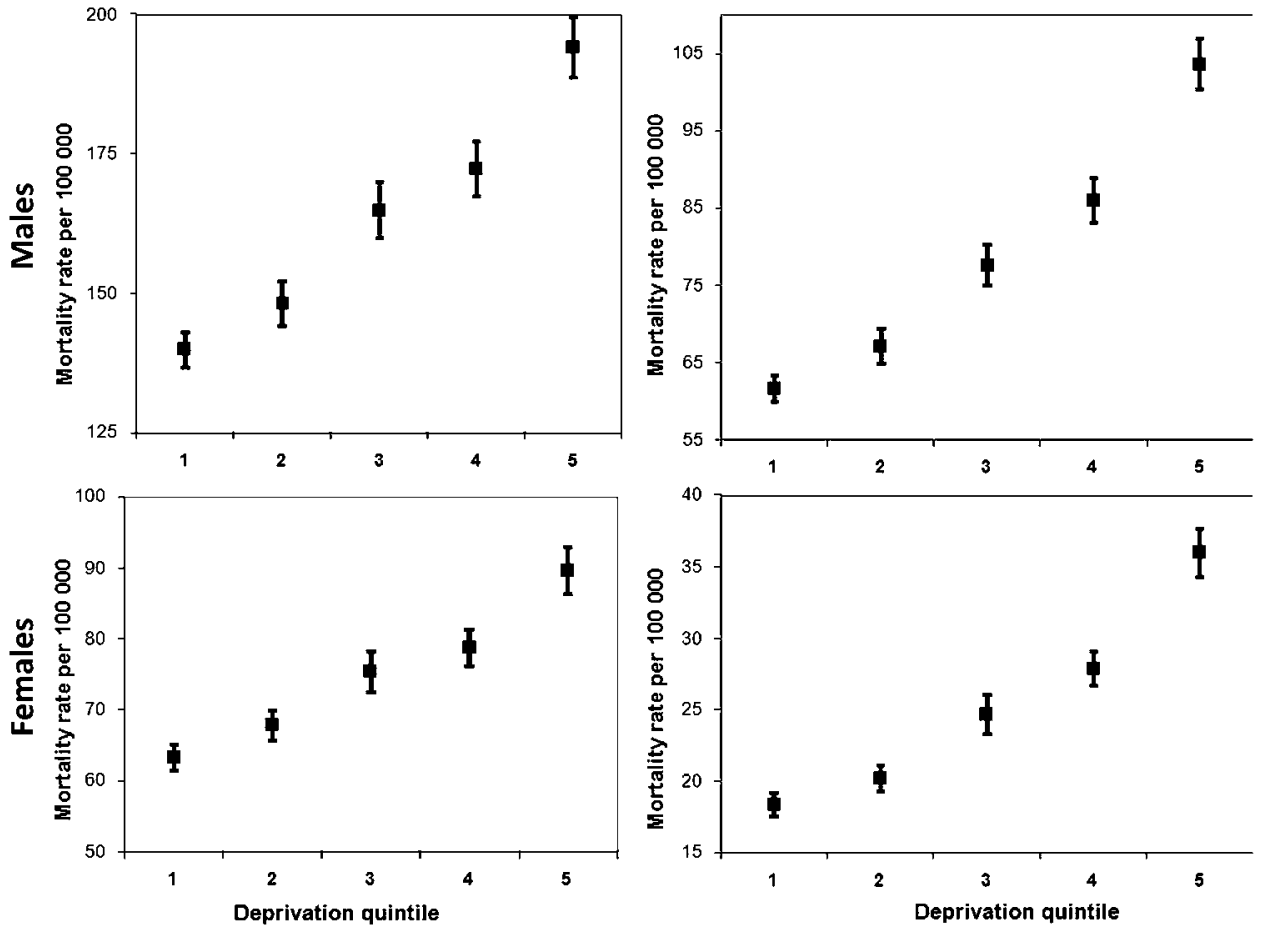

All ages

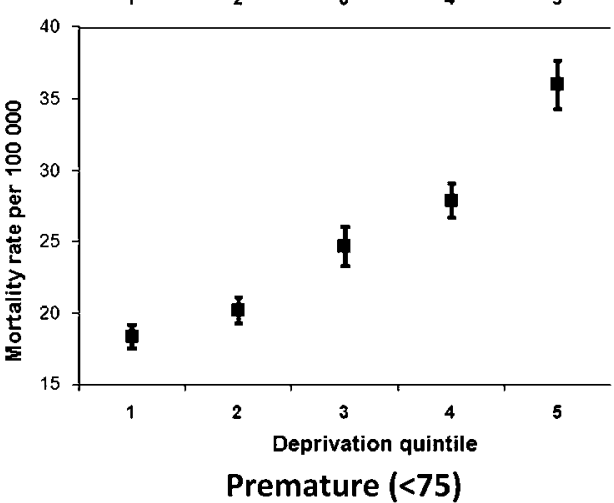

strongly associated with mortality rates in univariate analyses and significantly associated with mortality rates in multivariate analyses. Taking premature mortality among men (aged $<75$ years) as an example, the $\beta$ coefficient from univariate models shows that a 1-point increase in absolute deprivation is associated with an increase of 4.3 deaths per 100000 in the CHD mortality rates and that a 1-point increase in deprivation inequality is associated with an increase of 6.2 deaths per 100000 in $\mathrm{CHD}$ mortality rates. This suggests that the association between absolute deprivation and deprivation inequality and CHD mortality rates was independent of each other. Table 3 shows that the association with absolute deprivation was stronger than that with deprivation inequality-this is demonstrated by the attenuation of the $\beta$ coefficient for deprivation inequality (eg, in the male premature mortality models, the $\beta$ coefficients for deprivation inequality were 6.2 in the univariate model and 1.6 in the multivariate model). In each of the models, the spatial error variable was significantly associated with mortality rates, indicating the presence of spatial autocorrelation.

Table 2 Correlation coefficients between LA-level dependent and independent variables

\begin{tabular}{|c|c|c|c|c|c|}
\hline & $\begin{array}{l}\text { Male } \\
\text { mortality } \\
\text { rate }\end{array}$ & $\begin{array}{l}\text { Female } \\
\text { mortality } \\
\text { rate }\end{array}$ & $\begin{array}{l}\text { Male } \\
\text { premature } \\
\text { mortality } \\
\text { rate }\end{array}$ & $\begin{array}{l}\text { Female } \\
\text { premature } \\
\text { mortality } \\
\text { rate }\end{array}$ & $\begin{array}{l}\text { Absolute } \\
\text { deprivation }\end{array}$ \\
\hline $\begin{array}{l}\text { Female } \\
\text { mortality rate }\end{array}$ & 0.92 & & & & \\
\hline $\begin{array}{l}\text { Male } \\
\text { premature } \\
\text { mortality rate }\end{array}$ & 0.94 & 0.84 & & & \\
\hline $\begin{array}{l}\text { Female } \\
\text { premature } \\
\text { mortality rate }\end{array}$ & 0.88 & 0.90 & 0.91 & & \\
\hline $\begin{array}{l}\text { Absolute } \\
\text { deprivation }\end{array}$ & 0.62 & 0.55 & 0.77 & 0.72 & \\
\hline $\begin{array}{l}\text { Deprivation } \\
\text { inequality }\end{array}$ & 0.50 & 0.46 & 0.56 & 0.53 & 0.58 \\
\hline
\end{tabular}

All correlations were significant at $p<0.001$.
Table 4 shows the results of the ward-level regression analyses. Both absolute deprivation and relative deprivation were significantly associated with CHD mortality rates in all univariate models. Taking premature mortality among men (aged $<75$ years) as an example, the $\beta$ coefficient from univariate models shows that a 1-point increase in absolute deprivation is associated with an increase of 6.5 deaths per 100000 in CHD mortality rates and that a 1-point increase in relative deprivation is associated with an increase of 6.9 deaths per 100000 in CHD mortality rates. Combined multivariate models showed that relative deprivation was significantly associated with $\mathrm{CHD}$ mortality rates even after adjustment for absolute deprivation. The adjusted associations between relative deprivation and CHD mortality rates were not particularly strong, and inclusion of relative deprivation in the multivariate models did very little to improve the explanatory power of the models (eg, adding relative deprivation to the model of male $\mathrm{CHD}$ mortality rates and absolute deprivation only reduced the sum of the residuals from 2408 to 2407 and the $r^{2}$ remained unchanged at 0.41). Subgroup analyses showed that the independently significantly positive association between relative deprivation and CHD mortality rates was apparent when analyses were restricted to only affluent or only deprived wards. For each of the outcome variables in the subgroup analyses (male and female all ages CHD mortality rates, and male premature $\mathrm{CHD}$ mortality rates), the size of the association between relative deprivation and mortality rates after adjustment for absolute deprivation was very similar to the results from the main analyses (results not shown).

\section{DISCUSSION}

The results show that deprivation inequality is associated with CHD mortality rates for LAs in England, even after adjustment for the absolute level of deprivation of the LAs. This would be expected whether deprivation inequality within an LA had an impact on individual-level mortality risk or not. But further, the relative deprivation of a ward in comparison to its neighbours is associated with CHD mortality rates after adjustment for the 
Table 3 Spatial error regression models for CHD mortality rate and LA deprivation, and CHD mortality rate and variation in LA deprivation (measured as the IQR of within-LA deprivation scores), and of both combined (LAs, $\mathrm{n}=352$ )

\begin{tabular}{|c|c|c|c|c|}
\hline & Residual & $\begin{array}{l}\text { Absolute deprivation } \\
\beta(95 \% \mathrm{CI})\end{array}$ & $\begin{array}{l}\text { Deprivation inequality } \\
\beta(95 \% \mathrm{CI})\end{array}$ & Adjusted $r^{2}$ \\
\hline \multicolumn{5}{|c|}{ Men, all ages } \\
\hline Model A & 388 & 4.84 (4.21 to 5.47$)$ & & 0.40 \\
\hline Model B & 477 & & 7.67 (6.26 to 9.08 ) & 0.26 \\
\hline Model C & 377 & 4.06 (3.29 to 4.84$)$ & 2.70 (1.14 to 4.26$)$ & 0.42 \\
\hline \multicolumn{5}{|c|}{ Women, all ages } \\
\hline Model A & 122 & 2.24 (1.89 to 2.60$)$ & & 0.31 \\
\hline Model B & 137 & & 3.79 (3.04 to 4.55$)$ & 0.23 \\
\hline Model C & 118 & 1.75 (1.32 to 2.19 ) & 1.71 (0.84 to 2.57 ) & 0.34 \\
\hline \multicolumn{5}{|c|}{ Men, $<75$ years } \\
\hline Model A & 137 & 4.26 (3.88 to 4.63 ) & & 0.57 \\
\hline Model B & 220 & & 6.18 (5.22 to 7.13$)$ & 0.31 \\
\hline Model C & 133 & 3.78 (3.32 to 4.24$)$ & 1.61 (0.68 to 2.54 ) & 0.58 \\
\hline \multicolumn{5}{|c|}{ Women, $<75$ years } \\
\hline Model A & 30 & 1.72 (1.54 to 1.89$)$ & & 0.49 \\
\hline Model B & 42 & & 2.60 (2.18 to 3.02 ) & 0.29 \\
\hline Model C & 29 & 1.47 (1.25 to 1.68$)$ & 0.85 (0.42 to 1.28$)$ & 0.51 \\
\hline
\end{tabular}

Spatial error variable was significantly positively associated with the outcome variable in each model, indicating positive spatial autocorrelation. 'Absolute deprivation' refers to LA-level deprivation; 'Deprivation inequality' refers to IQR of ward-level deprivation scores for each $L A$; model $A$ and model $B$ are univariate models, model $C$ is a multivariate model.

CHD, coronary heart disease; LAs, local authorities.

absolute deprivation of the ward. This was shown in analyses of all wards and in subgroup analyses of affluent and deprived wards, which suggests that poor wards surrounded by rich wards have worse CHD mortality rates than poor wards surrounded by other poor wards and also that rich wards surrounded by poor wards have worse CHD mortality rates than rich wards surrounded by rich wards. Since the ward-level results do not include any measures of the distribution of deprivation within the wards they are not vulnerable to the statistical artefact that relations are driven by individual-level associations between absolute deprivation and health, which could generate spurious associations between inequality and health outcomes. ${ }^{7}$ The finding therefore provides evidence that deprivation inequality is adversely associated with CHD mortality for both rich and poor individuals within a society.

The practical importance of these findings relates to the difference in impact of redistributive policies that are suggested by the two competing hypotheses discussed earlier. It follows from the numerous studies of the association between income inequality and mortality rates that reducing social inequalities within a society would improve the health outcomes of the society as a whole. Under the first hypothesis (supported by the results of this paper), where the level of social inequality in society has an impact on all individuals within the society, any potential negative impact of redistributive policies on the rich within society should be balanced by the positive impact of reduction in social inequality, so it is conceivable that redistributive policies could have positive health consequences for both the poor and rich within society. That is, the richer may experience better health outcomes in a more equal society because the access to, and overall quality of, health goods and services improves as inequality lessens.

Under the competing hypothesis, redistributive policies will inevitably have negative health consequences on the rich within society, although at a population level, these will be outweighed by the positive health consequences for the poor. The results

Table 4 Regression models for CHD mortality rate and absolute deprivation, and CHD mortality rate and relative deprivation (measured as difference between deprivation and mean deprivation of neighbouring wards), and of both combined (wards, $\mathrm{n}=7929$ )

\begin{tabular}{|c|c|c|c|c|}
\hline & Residual & $\begin{array}{l}\text { Absolute deprivation } \\
\beta(95 \% \mathrm{CI})\end{array}$ & $\begin{array}{l}\text { Relative deprivation } \\
\beta(95 \% \mathrm{CI})\end{array}$ & Adjusted $r^{2}$ \\
\hline \multicolumn{5}{|c|}{ Men, all ages } \\
\hline Model A & 3714 & 7.66 (7.40 to 7.93$)$ & & 0.29 \\
\hline Model B & 4265 & & 8.61 (7.87 to 9.35$)$ & 0.06 \\
\hline Model C & 3710 & 7.43 (7.14 to 7.72$)$ & $1.42(0.72$ to 2.12$)$ & 0.29 \\
\hline \multicolumn{5}{|c|}{ Women, all ages } \\
\hline Model A & 2202 & 3.50 (3.34 to 3.65$)$ & & 0.19 \\
\hline Model B & 2392 & & 4.28 (3.87 to 4.70$)$ & 0.05 \\
\hline Model C & 2199 & 3.32 (3.15 to 3.50$)$ & 1.07 (0.65 to 1.48$)$ & 0.20 \\
\hline \multicolumn{5}{|c|}{ Men, $<75$ years } \\
\hline Model A & 2408 & 6.52 (6.35 to 6.69$)$ & & 0.41 \\
\hline Model B & 3013 & & 6.91 (6.39 to 7.44 ) & 0.08 \\
\hline Model C & 2407 & $6.40(6.22$ to 6.59$)$ & $0.72(0.26$ to 1.17$)$ & 0.41 \\
\hline
\end{tabular}

'Absolute deprivation' refers to ward-level deprivation; 'Relative deprivation inequality' refers to modulus of the difference between deprivation in the ward and the average deprivation in all neighbouring wards; model $\mathrm{A}$ and model $\mathrm{B}$ are univariate models, model $\mathrm{C}$ is a multivariate model.

$\mathrm{CHD}$, coronary heart disease. 
presented here suggest that, with regard to $\mathrm{CHD}$, it is possible to produce redistributive policies that would have positive health consequences for both rich and poor; however, the weak strength of the association between relative deprivation and CHD mortality rates after adjustment for absolute deprivation suggests that such policies would necessarily have a very limited impact. Future work could be directed at modelling the impact of introducing redistributive policies that have been designed to have positive health consequences for all individuals within a society. This paper presents cross-sectional data, and caution should be taken in inferring causality when considering either of the hypotheses presented above. Longitudinal analysis, possibly involving natural experiments, would be necessary to confirm causal relationships.

This study uses data on 241315 CHD deaths among men and 200689 CHD deaths among women in England between 2001 and 2006 and allows for the analysis of the impact of deprivation inequality for small areas in England. To our knowledge, the method developed to assess the impact of relative deprivation at ward level has not been applied elsewhere. We also think that this is the first time that such an analysis has been conducted with areas as small as wards. However, a limitation of the study is a lack of individual-level mortality data, which limits the effectiveness of the study design to assess whether area-level deprivation inequalities have a direct impact on individual health outcomes. Equally, it would be valuable to include individual-level data on variables that potentially confound or disguise the association between relative inequalities on health, such as migration between wards. ${ }^{16}$ By focusing on small areas, we have tried to maximise the impact of deprivation inequality on CHD mortality rates. This is because area-level effects on individuals tend to have larger impacts when measured at a smaller area level; people tend to identify their neighbourhood with only a small area around where they live; populations within small areas tend to be more homogeneous than populations within larger areas. Usually, analyses of the impact of social inequality on health are conducted using relatively large areas such as countries or, in the case of the US, states. There is potential with such large areas for confounding of the relationship between inequality and health because of possible variation in equality at smaller levels than that typically used for comparison. ${ }^{17}$ This is not an issue for this study, where the areas of interest (LAs and wards) are too small to have control over welfare and health policies that could have appreciable effects on their populations.

Other potentially explanatory area-level variables were not included in this study including heterogeneity within LAs (some are wholly urban, others wholly rural and others still a mix of urban and rural). Relationships between 'urbanicity' and CHD are well established, and the level of urbanisation within LAs may explain some of the variance observed in our study. Urbanisation is itself a proxy measure of other things, such as population density, access to health services, and so on, and so cannot specifically cause disease. Similarly, the deprivation index used here is a proxy of social position, and it is not clear to what degree it produces a scale of social position that is meaningful to the hypotheses explored here. Inaccuracies introduced by the deprivation index will lead to misspecification of the areas, which is likely to bias estimates to the null hypothesis-if this is the case, then the impact of relative deprivation reported here may be under-estimated.

A previous study ${ }^{18}$ has used area-level data to assess whether the relationship between income inequality and mortality rates can be entirely explained by the statistical artefact identified by

\section{What is already known on this subject}

Social position has a large and well-documented impact on many health outcomes.

- There are two competing hypotheses: that within developed high-income societies, the total amount of social inequality increases the risk of poor health outcomes for all individuals within the society and that an individual's social position is associated with individual-level adverse health outcomes, but the total amount of social inequality within society is not.

\section{What this study adds}

- The results show that deprivation inequality is associated with coronary heart disease mortality rates for local authorities in England, even after adjustment for the absolute level of deprivation of the local authorities.

- The paper suggests that poor wards surrounded by rich wards have worse coronary heart disease mortality rates than poor wards surrounded by other poor wards and also that rich wards surrounded by poor wards have worse coronary heart disease mortality rates than rich wards surrounded by rich wards.

- The finding provides evidence that deprivation inequality is adversely associated with coronary heart disease mortality for both rich and poor individuals within a society.

Gravelle. ${ }^{7}$ The researchers created a hypothetical data set where all-cause mortality rates within US states were entirely due to individual-level income of the population within the states and then showed that these data showed a positive association between state-level income inequality and mortality rates (demonstrating the statistical artefact). Crucially, the positive association was smaller using hypothetical data than when the equivalent genuine data were used, indicating that not all the relationships between income inequality and mortality rates were due to the statistical artefact. The results here extend that conclusion to small areas within England. The results of these analyses also concur with a recent meta-analysis of multilevel studies that concluded that income inequality has a small but significant association for all-cause mortality, after adjustment for individual-level income. ${ }^{5}$ Additional information offered by this study includes the confirmation that the impact of deprivation inequality on health outcomes can have an impact at area levels as small as wards.

Competing interests None.

Provenance and peer review Not commissioned; externally peer reviewed.

\section{REFERENCES}

1. Marmot M, Allen J, Goldblatt P, et al. Fair society, healthy lives: a strategic review of health inequalities in England post-2010. London: The Marmot Review, 2010.

2. Wilkinson RG. Income distribution and life expectancy. BMJ 1992;304:165-8.

3. Kondo N, Kawachi I, Subramanian SV, et al. Do social comparisons explain the association between income inequality and health? Relative deprivation and perceived health among male and female Japanese individuals. Soc Sci Med 2008:67:982-7.

4. Wilkinson RG, Pickett KE. Income inequality and socioeconomic gradients in mortality. Am J Public Health 2008;98:699-704.

5. Kondo N, Sembajwe G, Kawachi I, et al. Income inequality, mortality, and self-rated health: meta-analysis of multilevel studies. BMJ 2009;339:4471. 
6. Wilkinson RG. Socioeconomic determinants of health: Health inequalities: relative or absolute material standards? BMJ 1997;314:591.

7. Gravelle H. How much of the relation between population mortality and unequal distribution of income is a statistical artefact? BMJ 1998;316:382-5.

8. Lynch J, Smith GD, Harper S, et al. Is income inequality a determinant of population health? Part 1. A systematic review. Milbank 0 2004;82:5-99.

9. Mellor JM, Milyo J. Income inequality and population health: evidence from the current population survey. Health Serv Res 2002;38:137-52.

10. Ben-Shlomo Y, White IR, Marmot M. Does the variation in the socioeconomic characteristics of an area affect mortality? BMJ 1996;312:1013-14.

11. MIMAS. Casweb. 2001 Census Data for England and Wales. Manchester: University of Manchester. http://casweb.mimas.ac.uk (accessed 2008).
12. Carstairs V, Morris R. Deprivation and health in Scotland. Health Bull (Edinb) 1990;48:162-75

13. Morgan 0, Baker A. Measuring deprivation in England and Wales using 2001 Carstairs scores. Health Stat 0 2006:31:28-33.

14. Cliff A, Ord J. Spatial Autocorrelation. London: Pion, 1973.

15. Anselin L. GeoDa Version 0.9. Illinois: University of Illinois, 2003.

16. Subramanian S, Jones K, Kaddour A, et al. Revisiting Robinson: the perils of individualistic and ecologic fallacy. Int J Epidemiol 2009;38:342-60.

17. Davey Smith G. Editorial: Income inequality and mortality: why are they related? BMJ 1996;312:987-8.

18. Wolfson M, Kaplan G, Lynch J, et al. Relation between income inequality and mortality: empirical demonstration. BMJ 1999;319:953-5. 